\title{
INFLUÊNCIA DA APLICAÇÃO DE VINHAÇA NA CAPACIDADE DE INFILTRAÇÃO DE UM SOLO DE TEXTURA FRANCO ARENOSA
}

\author{
Alexandre Barcellos Dalri ${ }^{1}$; Glauco Eduardo Pereira Cortez ${ }^{2}$; Luís Guilherme Senarese \\ Riul $^{3}$; Jairo Augusto Campos Araújo ${ }^{4}$, Raimundo Leite Cruz ${ }^{5}$. \\ ${ }^{I}$ Departamento de Ciências Agrárias, Centro Universitário Moura Lacerda, Ribeirão Preto, SP, \\ abdalri@uol.com.br \\ ${ }^{2}$ Departamento de Ciências Exatas, Universidade Estadual Paulista, Campus de Jaboticabal, SP, \\ glauco@fcav.unesp.br \\ ${ }^{3}$ CERP - Centrais Energéticas de Ribeirão Preto, Ribeirão Preto, SP, lgriul@yahoo.com.br \\ ${ }^{4}$ Departamento de Engenharia Rural, Universidade Estadual Paulista, Campus de Jaboticabal, SP, \\ jaca@fcav.unesp.br \\ ${ }^{5}$ Departamento de Engenharia Rural, Universidade Estadual Paulista, Campus de Botucatu, SP, \\ cruz@fca.unesp.br
}

\section{RESUMO}

Este trabalho teve como objetivo estudar o efeito da aplicação de diferentes lâminas de vinhaça na infiltração de água no solo e verificar a adequação dos modelos de Horton e Kostiakov-Lewis na estimativa da taxa de infiltração. Os tratamentos corresponderam à aplicação de cinco doses de vinhaça $\left(0,200,250,300\right.$ e $\left.350 \mathrm{~m}^{3} \cdot \mathrm{ha}^{-1}\right)$. A aplicação de vinhaça propiciou uma redução da taxa de infiltração básica do respectivo solo em estudo, causando efeito negativo para essa característica, pois, eleva o risco de erosão do solo e majora a possibilidade do escoamento superficial. O modelo de Horton propiciou o melhor ajuste sobre os dados observados, e o modelo de Kostiakov-Lewis superestimou os valores da taxa de infiltração.

UNITERMOS: vinhaça, infiltração, solo.

DALRI, A.B.; CORTEZ, G.E.P.; RIUL, L.G.S.; ARAÚJO, J.A.C.; CRUZ, R.L. VINASSE APPLICATION INFLUENCE ON INFILTRATION CAPACITY OF SANDY LOAM SOIL

\section{ABSTRACT}

The objective of this study was to analyze effects in different depth in soil water infiltration and to verify the Horton and Kostiakov-Lewis models adequacy in infiltration rate estimate. The treatments were five doses of vinasse $\left(0,200,250,300\right.$ and $\left.350 \mathrm{~m}^{3} \cdot \mathrm{ha}^{1}\right)$. The vinasse application reduced the soil erosion risk increasing the possibility of runoff. The Horton model had the best adjustment on the observed data, and the Kostiakov-Lewis model overestimated the infiltration rate values.

KEYWORDS: vinasse, infiltration, soil. 


\section{INTRODUÇÃO}

A infiltração é definida como a passagem de água da superfície para o interior do solo, constituindo-se num dos mais importantes processos que compõem o ciclo hidrológico por ser fator determinante da disponibilização de água para as culturas, da recarga dos aqǘferos subterrâneos, da ocorrência e magnitude do escoamento superficial e do manejo do solo e da água. O escoamento superficial é responsável por processos indesejáveis, como a erosão do solo e as inundações, logo a infiltração determina o balanço de água na zona radicular e, por isso, o conhecimento desse processo e suas relações com as propriedades do solo é de fundamental importância para o eficiente manejo do solo e da água (Brandão et al., 2002).

O conhecimento do processo de infiltração fornece subsídios para o dimensionamento de reservatórios, estruturas de controle de erosão e de inundação, canais e sistemas de irrigação e drenagem (Brandão et al., 2002), e é essencial para o sucesso da modelagem hidrológica de bacias hidrográficas (Riguetto, 1998).

O processo de infiltração de água no solo pode ser descrito por diversas equações ou modelos, alguns desenvolvidos a partir de considerações físicas, enquanto outros são de forma empírica. Um modelo empírico muito empregado em manejo de irrigação é a equação de Kostiakov-Lewis, normalmente utilizadado para a estimativa da infiltração acumulada, cujos parâmetros não têm significado físico próprio e são estimados a partir de dados experimentais. O modelo de Horton, o qual também é um modelo empírico, porém considerado mais consistente que o de Kostiakov-Lewis, é escrito na forma de uma função exponencial. Segundo Prevedello (1996), no modelo de Horton a redução de infiltração com o tempo é fortemente controlada por fatores que agem na superfície do solo, tais como selamento superficial, fenômenos de expansão e contração do solo.

Solos de textura grossa, ou seja, solos arenosos possuem maior quantidade de macroporos do que solos de textura fina e, conseqüentemente apresentam maior condutividade hidráulica saturada e taxa de infiltração. Por outro lado, solos argilosos bem estruturados, ou com estrutura estável, podem apresentar maiores taxas de infiltração do que os solos com estrutura instável, que sofrem dispersão quando umedecidos ou submetidos a algum agente desagregador. A estabilidade dos agregados é determinada pelos chamados agentes cimentantes, que são representados principalmente pela matéria orgânica e pelos óxidos de ferro e alumínio. Dessa forma, na medida em que aumenta a presença destes compostos no solo, maior é a possibilidade deste apresentar uma estrutura mais estável (Shainberg \& Levy, 1995; Silva \& Kato, 1997). Em função de sua composição físicoquímica, a vinhaça comporta-se como agente cimentante do solo (Glória, 1975).

O processo clássico de produção de etanol derivado do caldo da cana-de-açúcar, bem como a produção de cachaça, gera como resíduo após a destilação, a vinhaça. A grande preocupação com a vinhaça advém basicamente da sua composição química que a torna um grande poluidor, em decorrência de suas características ácidas e corrosivas, se lançada sem tratamento apropriado aos meios hídricos.

Copersucar (1978) recomenda o emprego de vinhaça como fertilizante, pois repõem ao solo parte dos nutrientes que a planta dele retira, levando ao aumento da produtividade agrícola, elevação do $\mathrm{pH}$ do solo, aumento da disponibilidade de alguns nutrientes e imobilização de outros, aumento da população microbiana, aumento no poder de retenção de água e melhoria da estrutura física.

A adição prolongada de diferentes quantidades de vinhaça em quatro diferentes tipos de solo Latossólico, por períodos de até 20 anos não resultou em qualquer efeito prejudicial ao solo, como acúmulo de sais; pelo contrário, houve uma melhoria de suas propriedades 
químicas, traduzidas pela redução da acidez e elevação dos teores de potássio, cálcio e magnésio, além da soma de bases e da capacidade de troca catiônica efetiva do solo (Orlando Filho et al., 1983).

Segundo Silva et al. (2007), a bibliografia acerca dos efeitos da vinhaça no solo e nas águas subterrânea ainda é bastante limitada, e devido a essa deficiência, os resultados encontrados são muito variáveis em razão da grande diversidade de solos e composição das vinhaças.

Este trabalho teve como objetivo estudar o efeito da aplicação de diferentes lâminas de vinhaça na infiltração de água no solo e verificar a adequação dos modelos de Horton e Kostiakov-Lewis na estimativa da taxa de infiltração.

\section{MATERIAL E MÉTODOS}

O trabalho foi conduzido o Campus do Centro Universitário Moura Lacerda, em Ribeirão Preto, SP (latitude $21^{\circ} 48^{\prime} \mathrm{S}$, longitude $47^{\circ} 48^{\prime} \mathrm{W}$ e altitude de $546 \mathrm{~m}$ ). O solo onde foi realizado o ensaio apresenta textura franco arenosa. A cultura presente no local onde foram realizados os testes é citrus, sendo que nas entre linhas onde foram colocados os anéis predomina a brachiaria.

A área experimental possui declividade média de 1\%. E no mesmo local foram coletadas amostras de solo, as quais foram submetidas às seguintes determinações: densidade de partículas (dp), densidade do solo (ds), e textura. Os respectivos valores encontram-se na Tabela 1.

Tabela 1. Caracterização física do solo utilizado no presente estudo.

\begin{tabular}{ccccccc}
\hline $\begin{array}{c}\text { Profundidade } \\
(\mathrm{cm})\end{array}$ & Areia & Silte & $\begin{array}{c}\text { Argila } \\
(\%)\end{array}$ & Porosidade & Ds & \multicolumn{2}{c}{ Ds } \\
\hline $0-20$ & 70 & 4 & 26 & 35,06 & 1,76 & 2,71 \\
$20-40$ & 68 & 5 & 27 & 30,56 & 1,65 & 2,73 \\
\hline
\end{tabular}

A Tabela (2) apresenta as características físico-químicas da vinhaça utilizada no experimento.

Tabela 2. Análise físico-química da vinhaça utilizada no experimento.

\begin{tabular}{ccccc}
\hline $\begin{array}{c}\text { Nitrogênio } \\
\text { amoniacal } \\
\text { total }\end{array}$ & $\begin{array}{c}\text { Fósforo } \\
\mathrm{mg}^{-1}\end{array}$ & $\begin{array}{c}\text { Potássio } \\
\left(\mathrm{K}_{2} \mathrm{O}\right)\end{array}$ & $\mathrm{pH}$ & $\begin{array}{c}\text { Sólidos totais } \\
(\%)\end{array}$ \\
\hline 64,4 & 91,0 & 3120,0 & 4,48 & 0,80 \\
\hline
\end{tabular}

O experimento contou com cinco tratamentos $0,200,250,300$ e $350 \mathrm{~m}^{3} \cdot \mathrm{ha}^{-1}$ e quatro repetições.

Para a determinação da taxa de infiltração básica (TIB) de água no solo utilizou-se o método do infiltrômetro de anéis concêntricos. Adotou-se um intervalo de 48 horas entre as aplicações de vinhaça. Fez-se o teste com a dose zero, 48 horas depois houve a primeira aplicação de vinhaça, dose $200 \mathrm{~m}^{3} \cdot \mathrm{ha}^{-1}$, esperou-se um intervalo de 48 horas e fez-se o teste de infiltração e assim sucessivamente até a dose $350 \mathrm{~m}^{3}$.ha $\mathrm{a}^{-1}$. A vinhaça foi aplicada manualmente com o auxílio de um recipiente plástico. O intervalo fixo entre os testes de infiltração permite afirmar que o teor de água inicial no solo foi aproximadamente o mesmo. 
Os valores de taxa de infiltração foram obtidos pela relação entre lâmina infiltrada e o tempo de infiltração considerado.

Com os dados da taxa de infiltração média obtidos no campo, verificou-se a adequação dos modelos de Horton, Equação (1), e Kostiakov-Lewis, Equação (2), para a estimativa da taxa de infiltração de água nos diferentes manejos de aplicação de vinhaça.

$$
\begin{aligned}
& \mathrm{i}=\mathrm{i}_{\mathrm{f}}+\left(\mathrm{i}_{0}-\mathrm{i}_{\mathrm{f}}\right) \mathrm{e}^{-\beta \mathrm{t}} \\
& \mathrm{i}=\mathrm{k} \alpha \mathrm{t}^{(\alpha-1)}+\mathrm{i}_{\mathrm{f}}
\end{aligned}
$$

Em que:

$$
\begin{aligned}
& \mathrm{i}_{\mathrm{o}}=\text { taxa de infiltração inicial observada, } \mathrm{cm} \cdot \mathrm{h}^{-1} \text {; } \\
& \mathrm{i}_{\mathrm{f}}=\text { taxa de infiltração final, } \mathrm{cm} \cdot \mathrm{h}^{-1} ; \\
& \alpha, \beta \text { e } \mathrm{k}=\text { parâmetros estatísticos, } \mathrm{e} \\
& \mathrm{t}=\text { tempo de infiltração, min. }
\end{aligned}
$$

Os parâmetros $\alpha, \beta$, e k, foram estimados utilizando programa computacional Microcal Origin ${ }^{\circledR}$ versão 6.0. A qualidade dos ajustes dos modelos foi avaliada por meio de regressões não lineares entre os valores estimados e os valores médios observados em cada tratamento estudado, juntamente com os respectivos coeficientes de determinação. Nessa avaliação também foi utilizado os índices estatísticos: coeficiente de massa residual (CMR), coeficiente de ajuste (CA) e eficiência (EF), representados, respectivamente pelas Equações (3), (4) e (5).

$$
\begin{gathered}
C M R=\frac{\left(\sum_{i=1}^{n} O i-\sum_{i=1}^{n} P i\right)}{\sum_{i=1}^{n} O i} \\
C A=\frac{\sum_{i=1}^{n}(O i-\bar{O})^{2}}{\sum_{i=1}^{n}(P i-\bar{O})^{2}} \\
E F=\frac{\left[\frac{\left[\sum_{i=1}^{n}(O i-\bar{O})^{2}\right.}{\left.\sum_{i=1}^{n}(O i-\bar{P})^{2}\right]}\right.}{\sum_{i=1}^{n}(O i-\bar{O})^{2}}
\end{gathered}
$$

Em que:

$\mathrm{Oi}=$ valores observados;

$\mathrm{Pi}=$ valores estimados;

$\mathrm{n}=$ números de observações;

$\bar{O}=$ média aritmética das observações, e 
$\bar{P}=$ média aritmética dos valores estimados.

\section{RESULTADOS E DISCUSSÃO}

Nas condições naturais, sem aplicação de vinhaça, o solo em estudo apresentou taxa de infiltração básica (TIB) de 12,0 cm.h ${ }^{-1}$ (Figura 1). Quando a lâmina de vinhaça aplicada foi de $250 \mathrm{~mm}$, a TIB reduziu para $4,8 \mathrm{~cm} \cdot \mathrm{h}^{-1}$ (Figura 3). Provavelmente, essa redução foi causada pelo selamento inicial da camada superficial do solo devido à aplicação de vinhaça. Com as aplicações de doses de vinhaça equivalente a $300 \mathrm{~mm}$ e $350 \mathrm{~mm}$, a TIB encontrada foi de 7,2 e $6,0 \mathrm{~cm} \cdot \mathrm{h}^{-1}$, (Figuras 4 e 5). Observa-se que os valores foram inferiores à condição de solo sem aplicação de vinhaça.

De acordo com Camargo et al. (1983), o alto teor de matéria-orgânica presente na vinhaça propicia a mesma uma ação cimentante, agregando partículas pequenas em partículas maiores, elevando a TIB. Entretanto, para o solo arenoso estudado, a vinhaça não elevou a taxa infiltração de água do solo.

Em relação aos valores obtidos por meio do modelo de Kostiakov-Lewis, observa-se nas Figuras 1 a 5 que a partir dos dez primeiros minutos iniciais da aplicação de água, os valores simulados pela equação de Kostiakov-Lewis tendem a superestimar os valores ajustados. Resultado também encontrado por (Alves Sobrinho et al. 2003).

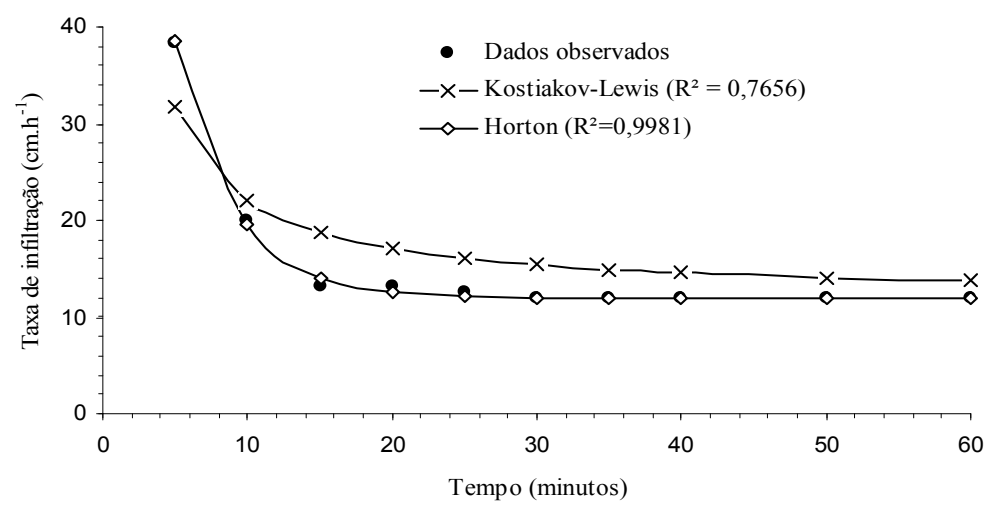

Figura 1. Taxas de infiltração observada e ajustada com os modelos para dose $0\left(\mathrm{~m}^{3} \cdot h \mathrm{~h}^{-1} \mathrm{de}\right.$ vinhaça).

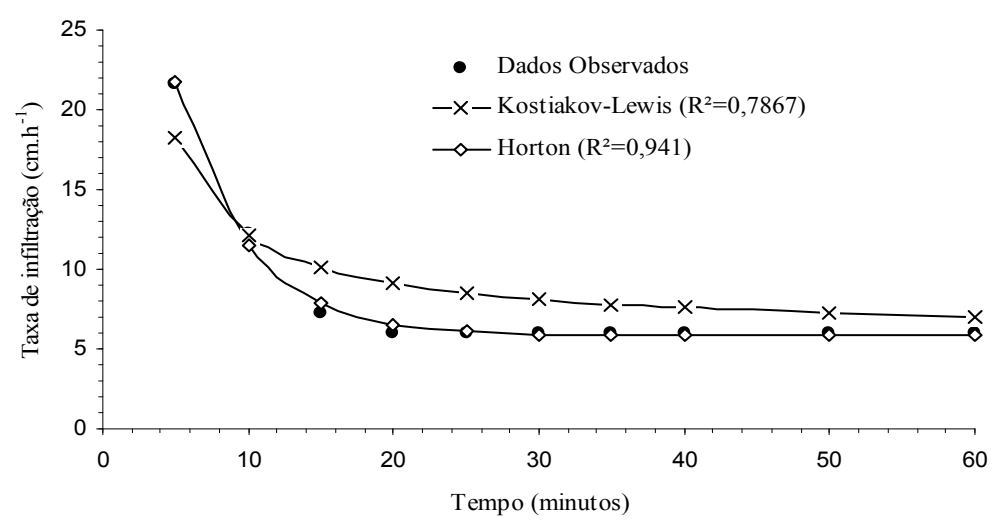

Figura 2. Taxas de infiltração observada e ajustada com os modelos para dose $200\left(\mathrm{~m}^{3} \cdot \mathrm{ha}^{-1}\right.$ de vinhaça). 


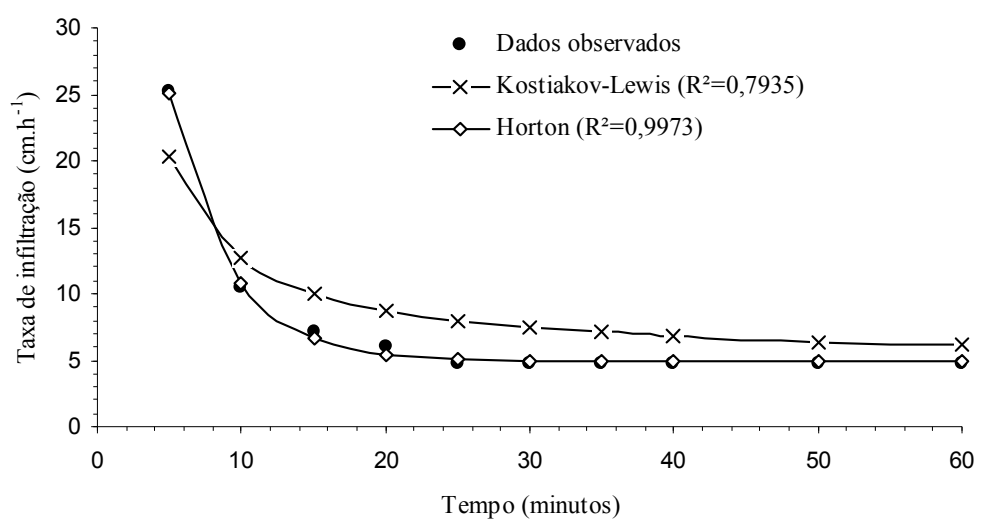

Figura 3. Taxas de infiltração observada e ajustada com os modelos para dose $250\left(\mathrm{~m}^{3} \cdot \mathrm{ha}^{-1} \mathrm{de}\right.$ vinhaça).

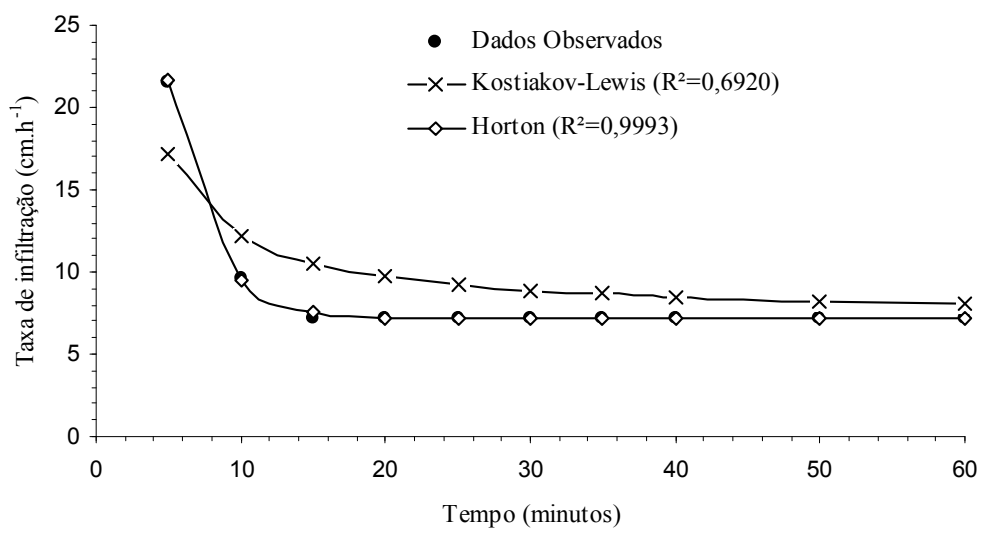

Figura 4. Taxas de infiltração observada e ajustada com os modelos para dose $300\left(\mathrm{~m}^{3} \cdot \mathrm{ha}^{-1}\right.$ de vinhaça).

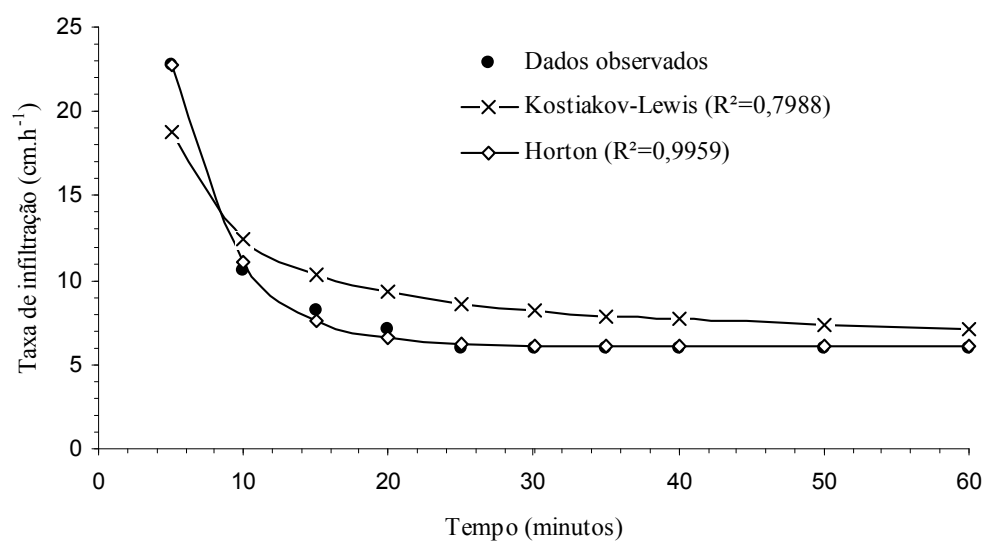

Figura 5. Taxas de infiltração observada e ajustada com os modelos para dose $350\left(\mathrm{~m}^{3} \cdot \mathrm{ha}^{-1} \mathrm{de}\right.$ vinhaça). 
Observa-se nas Figuras de 1 a 5, que o modelo de Horton propiciou ótimo ajuste com os dados observados, sendo esta satisfatória. Tal afirmação se baseia na análise dos coeficientes de determinação $\left(\mathrm{r}^{2}\right)$ das regressões não-lineares ajustadas em cada tratamento estudado, pois o modelo de Horton apresentou valores acima de 0,9410. Pelos ajustes realizados pela equação de Kostiakov-Lewis, não houve uma qualidade satisfatória, pois as análises dos coeficientes de determinação $\left(\mathrm{r}^{2}\right)$ das regressões não-lineares ajustadas apresentaram valores entre 0,6920 e 0,8591 , ou seja valores menores do que na equação proposta por Horton.

A curva de Kostiakov-Lewis superestimou os dados da taxa de infiltração básica (TIB) em todos os tratamentos estudados, apresentando valores da TIB superiores aos valores dos dados observados no campo. Analisando a infiltração de água no solo em sistemas de plantio direto e convencional, Alves Sobrinho et al. (2003) também encontraram melhor ajuste na equação de Horton, com valores do coeficiente de determinação $\left(\mathrm{r}^{2}\right)$ acima de 0,91 ao passo que a equação de Kostiakov-Lewis, superestimou os valores ajustados.

De acordo com Paixão et al. (2004), o modelo apresentado por Horton também foi o que apresentou melhor desempenho na estimativa dos valores médios da taxa de infiltração quando comparado com os demais modelos empíricos.

A Tabela 3 apresenta os parâmetros estatísticos $\alpha, \beta$, k, e os valores de infiltração inicial e final, $i_{\mathrm{o}}$ e $\mathrm{i}_{\mathrm{f}}$, respectivamente, para as Equações (1 e 2).

A Tabela 4 apresenta a relação dos índices estatísticos determinados pelas Equações (4, 5 e 6). Analisando o coeficiente de massa residual (CMR), verifica-se que a equação de Horton estima com boa exatidão a taxa de infiltração básica, pois os valores de (CMR) estão próximo ao índice zero ou mesmo são iguais a zero nos tratamentos com doses de 250, 300 e $350 \mathrm{~m}^{3} \cdot \mathrm{ha}^{-1}$ de vinhaça. Enquanto o modelo de Kostiakov-Lewis superestima os valores da $\mathrm{TIB}$, comportamento indicado pelos valores negativos do índice (CMR).

Tabela 3. Parâmetros de ajuste dos modelos

Tratamentos

\begin{tabular}{cccccc}
\hline \multicolumn{7}{c}{0} & 200 & 250 & 300 & 350 \\
$\beta$ & 0,2499 & 0,2056 & 0,2446 & 0,3645 & 0,2408 \\
$\mathrm{I}_{0}$ & 92,7079 & 68,7596 & 68,7596 & 89,4390 & 55,4258 \\
$\mathrm{I}_{\mathrm{f}}$ & 11,8949 & 4,8925 & 4,8925 & 7,1562 & 6,0947 \\
\multicolumn{5}{c}{ Modelo de Horton } \\
$\alpha$ & 0,0160 & 0,0169 & 0,0169 & 0,01301 & 0,01724 \\
$\mathrm{k}$ & 6184,2489 & 4462,1311 & 4462,1311 & 3753,4420 & 3620,1688 \\
\hline
\end{tabular}

O coeficiente de ajuste (CA) e a eficiência (EF) foram também melhores na equação de Horton, para os cinco tratamentos. Os valores do índice estatístico (CA), mais próximos da unidade, ratificam o modelo de Horton como o mais adequado para estimar a taxa de infiltração do respectivo solo estudado. Resultado semelhante foi obtido por Alves Sobrinho et al. (2003). Os valores de (CA) obtidos com os valores simulados pelo modelo de Horton, estão mais próximos da unidade em todos os tratamentos. 
Tabela 4. Índices estatísticos calculados

\begin{tabular}{lccccc}
\hline Índices & \multicolumn{5}{c}{ Tratamentos } \\
& 0 & 200 & 250 & 300 & 350 \\
\multicolumn{5}{c}{ Modelo de Kostiakov-Lewis } \\
CMR & $-0,1320$ & $-0,1558$ & $-0,2076$ & $-0,1406$ & $-0,1551$ \\
CA & 1,9770 & 1,6281 & 1,7509 & 1,7054 & 1,6544 \\
EF & 0,0015 & 0,040 & 0,0025 & 0,0050 & 0,0038 \\
& 0,0078 & $-0,0007$ & 0,0000 & 0,0000 & 0,0000 \\
CMR & 0,9907 & 1,0029 & 1,0010 & 1,0002 & 1,0013 \\
CA & 0,0016 & 0,0043 & 0,0027 & 0,0054 & 0,0040 \\
EF & \multicolumn{5}{c}{ Modelo de Horton } \\
\hline
\end{tabular}

\section{CONCLUSÕES}

A vinhaça propiciou redução da taxa de infiltração (TIB) do solo causando efeito negativo para essa característica. O modelo de Horton propiciou o melhor ajuste sobre os dados observados, e o modelo de Kostiakov-Lewis superestimou os valores da TIB.

\section{REFERÊNCIAS}

ALVES SOBRINHO, T. et al. Infiltração de água no solo em sistemas de plantio direto e convencional. Revista Brasileira de Engenharia Agrícola e Ambiental, Campina Grande, v. 7, n. 2, p. 191-196, 2003.

BRANDÃO, V. dos S. et al. Infiltração da água no solo. Viçosa: UFV, 2002. 98p.

CAMARGO, O. A.; VALADARES, J. M. A. S.; GERALDI, R. N. Características químicas e físicas de solo que recebeu vinhaça por longo tempo. Campinas: Instituto Agronômico, 1983. 30 p. (Boletim técnico, 76).

CAMBUIM, F. A. A ação da vinhaça sobre a retenção de umidade, $\mathbf{p H}$, acidez total, acumulação e lixiviação de nutrientes, em solo arenoso. 1983. 133 p. Dissertação (Mestrado em Ciências do Solo) - Universidade Federal Rural de Pernambuco, Recife, 1983.

COPERSUCAR - COOPERATIVA DE PRODUTORES DE CANA-DE-AÇÚCAR, AÇÚCAR E ÁLCOOL DO ESTADO DE SÃO PAULO. Aproveitamento da vinhaça: viabilidade técnico-econômica. Boletim Técnico Copersucar, Piracicaba, p. 1-66, 1978.

GLÓRIA, N. A. Utilização agrícola da vinhaça. Brasil Açucareiro, Piracicaba, v. 86, n. 5, p. 11-17, 1975. 
ORLANDO FILHO, J.; SILVA, G. M. A.; LEME, E. J. A. Utilização agrícola dos resíduos da agroindústria canavieira. In: PROGRAMA NACIONAL DE MELHORAMENTO DA CANA-DE-AÇÚCAR. Nutrição e adubação da cana-de-açúcar no Brasil. Piracicaba, 1983. p. 229-264. (Coleção Planalsucar, 2).

PAIXÃO, F. J. R. da et al. Estimativa da infiltração da água no solo através de modelos empíricos e funções não lineares. Revista de Biologia e Ciências da Terra, Campina Grande, v. 5, n. 1, 2004.

PREVEDELLO, C. L. Física do solo com problemas resolvidos. Curitiba: SAEAFS, 1996. $446 \mathrm{p}$.

RIGUETTO, A. M. Hidrologia e recursos hídricos. São Carlos: USP; EESC, 1998. 840 p.

SHAINBERG, I; LEVY, G. J. Infiltration and seal formation processes. In: AGASSI, M. (Ed.) Soil erosion, conservation, and rehabilitation. New York: Marcel Dekker, 1995. p. 122.

SILVA, C. L.; KATO, E. Efeito do selamento superficial na condutividade hidráulica saturada da superfície de um solo sob cerrado. Pesquisa Agropecuária Brasileira, Brasília, DF, v. 32, n. 2, p. 213-220, 1997.

SILVA, M. A. S. da; GRIEBELER, N. P.; BORGES, L. C. Uso de vinhaça e impactos nas propriedades do solo e lençol freático. Revista Brasileira de Engenharia Agrícola e Ambiental, Campina Grande, v. 11, n. 1, p. 108-114, 2007. 